\title{
VIABILITY-BASED REPLICATION MANAGEMENT SCHEME FOR RELIABLE IOT DATA SERVICES
}

\author{
Siwoo Byun* \\ Dept. of Software, Anyang University, Anyang-shi, \\ Kyonggi-do 430-714, South Korea \\ * swbyun@anyang.ac.kr
}

\begin{abstract}
This paper proposed a new replication management scheme for IoT sensor network environments. Replicating data across multiple nodes can increase the reliability for the shared data. However, data replication inevitably results in overhead of maintaining data consistency, especially in the event of excessive sensor node failures, requiring more costly synchronization mechanisms. In order to lessen the overhead of excessive node failures in fault-prone IoT sensor networks, a new scheme called sensor delegation con-sensus (SDC) was devised in this study. SDC minimizes the message exchange overheads of performing quorum access processes by means of viable deputy polling method which uses strong sensor nodes except unreliable nodes in polling process. This paper also proposed a computer simulation system to present the efficiency of SDC scheme. The performance evaluation results showed that the SDC model outperforms traditional replication management models.
\end{abstract}

Keywords - Frequent disconnection; IoT sensor network; Replication control; Weighted polling

\section{INTRODUCTION}

Recent development of mobile sensor devices has enabled the widespread deployment of Internet of Things (IoT) networks composed of sensor nodes (SN) (Figure 1). SNs have some resource constraints such as narrow network bandwidth, limited battery, small memory size, and weak processing power [1].

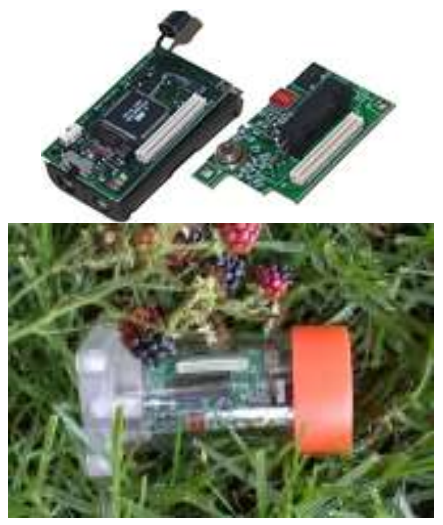

Fig. 1 Example of a device platform for IoT sensor network

Received: December 29, 2018

Reviewed: February 25, 2019

Accepted: March 5, 2019

* Corresponding Author 
The applications of IoT sensor networks in the future is varied and widespread. Much of the transmitted data is continuously monitored data and thus big sensor database is the critical component for IoT environment. That is, massive amounts of data detected by sensor nodes can generate various information, the sensor database will be the critical resource for the next IoT businesses.

In general, a special nodes called gateway(gw) are connected to nodes outside of the wireless sensor network through long-range networks such as internet, and thus all the communication with remote applications goes through the gw node or base station [2] in the sensor network as illustrated in Figure 2.

The performance of a sensor database could vary depending upon battery power capacity of sensor nodes and channel bandwidth in the wireless communication. Therefore, it is very critical to minimize the number of radio transmissions since large amounts of battery charges are required to project radio waves for each occurrence.

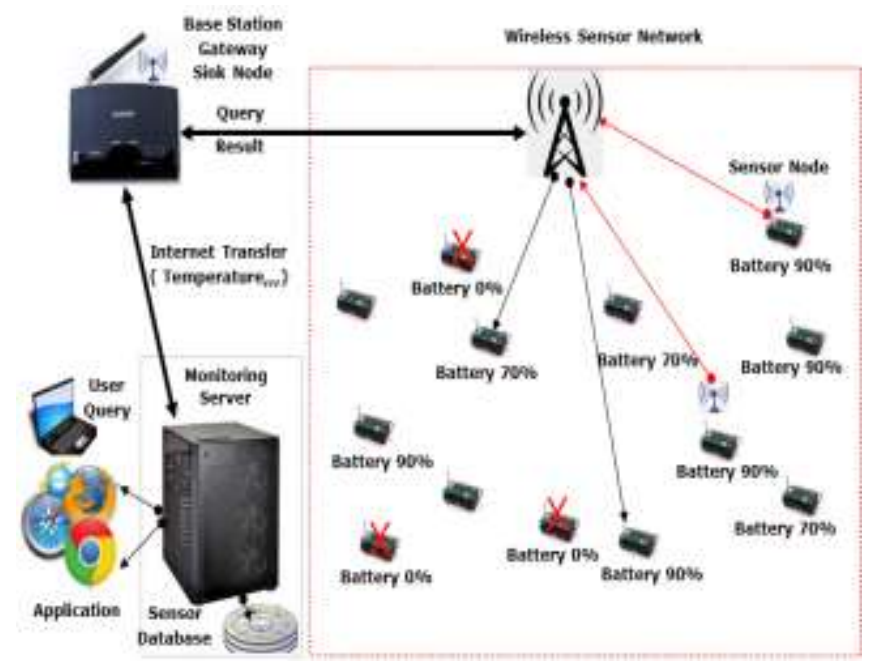

Fig. 2 Example of a wireless IoT sensor network

Another technical limitation related to the low bandwidth of the wireless media is that it often makes the local networks unavailable, especially in a high-contention sensor database environment. Moreover, since the bandwidth is shared among a number of SNs, the deliverable bandwidth per sensor nodes would be even lower. The unreliable wireless media is also vulnerable to various electronic interference that can provide unpredictable communication disruptions and communication errors.

\section{REPLICATION CONTROL FOR SENSOR NODES}

Because IoT sensor network has unreliable wireless channel and narrow bandwidth, the sensor-based applications could suffer from unreliable and ill-timed services. One way to reduce the likelihood of undesirable services is to replicate data across multiple SNs. The example of critical data replication may be a human location detected from body heats in disaster areas or battle fields.

In IoT sensor network environment, replication can contribute to high data availability in that each independent SN site can access its own data copy in case SN site(node) failures or wire-less communication failures last for a long time. Moreover, the actual operation cost of wireless communication is very expensive as comparted to the other operations(Table I)[3]. In this respect, data replication mechanism is far more important in the sensor network environment rather than in the general distributed network environment. 
Table I. Energy Consumption of Sensor Node Operations

\begin{tabular}{|c|c|}
\hline Sensor Node Operations & Ah(nano) \\
\hline \hline Packet transmission & 20.0 \\
\hline Packet Receiving & 8.00 \\
\hline Sensor Operation & 1.08 \\
\hline Accessing flash memory data & 5.51 \\
\hline ADC Sample Reading & 0.01 \\
\hline
\end{tabular}

Replicating data inevitably creates additional overhead of keeping copy consistency. All the replication control (RC) scheme aims to achieve both the replica consistency and high data availability.

However, current RC technologies are not yet considered to be sufficient to handle frequently disconnected sensor nodes in unreliable wireless network. In this respect, this paper concerned with this matter and proposed a new RC scheme.

The simplest scheme to manage replicated data is read-one write-all (ROWA)[4]. However, since write transactions cannot be allowed after one single node failure, ROWA naturally limits the data availability.

To cope with the low data availability problem, the polling(voting) algorithms [5,6] were proposed. The polling process requires write transactions to update a predefined number of copies (for example, [1/2+1]), instead of all copies in ROWA scheme.

The idea of polling has been generalized to general quorum-consensus (GQC) scheme [7]. In QC based schemes [8,9], replica-accessing transactions should formulate a quorum, a set of sites which has more than a certain predefined number, prior to actual read or write.

Quorum system is recognized as the main abstraction skill to ensure data consistency in fault-tolerant and reliable distributed databases [10]. Recently, QC algorithms have evolved to fault-tolerant event logger [11] and block chain system[12]

This paper also focused on these QC based schemes in the sense that they are efficient and reliable RC scheme which is able to tolerate both sensor node failures and network partitioning due to gateway failure.

However, if those schemes are applied to sensor network environment without any modification, large amount of message exchanges for polling process could lead to serious communication overhead and excessive battery consumption. Therefore, the overall transaction performance shall degrade substantially in the sensor network environment.

In practical cases, SNs may fail when their batteries are exhausted. For this case, QC scheme is used to achieve high fault-tolerance. However, replication process needs additional battery consumption; there exists a trade-off between energy-efficiency and fault-tolerance. Therefore, the replica with higher priority should be included first.

\section{OVERHEAD OF SENSOR ENVIRONMENTS}

In case the traditional QC based schemes are applied to sensor environment, the following overhead factors also need to be concerned in replication processes. 


\subsection{FREQUENT DISCONNECTIONS OF SENSOR NODES}

In general, distributed environment, the QC-based approach is considered as the most reliable approach since they can cope with both node failure and partitioning failure. In case there are sufficient operational sites to create a read or write quorum, e.g., more than half of the total sites, this excellent advantage of fault-tolerance can be preserved without any recovery procedure. Since the possibility that more than half of the total sites are down is very low, the QC based schemes are considered to be efficient and reliable in general distributed environment. However, this fault-tolerance feature of QC based schemes cannot be preserved in sensor network environment where SNs are essentially easy to be disconnected intentionally due to sleep mode or unpredictably due to weak WiFi strength. Example 1 shows this.

\section{Example 1. (Indefinite Delay Due to Frequent Disconnections):}

Suppose there are five copies for SNs at time t0. Thus, Vtotal is 5 and WT is 2.5. At t0, any quorum formulation is essentially al-lowed at every node site, and thus any transaction issued in each node is able to access x. Suppose that two sites, SN2 and SN3, failed at some time later, say $\mathrm{t} 1$, due to disconnection by their limited battery capacity. Note that replicated data of alive node is still serviceable despite the two sites failures in that $\{\mathrm{SN} 1, \mathrm{SN} 4, \mathrm{SN} 5\}$ is able to formulate write quorum. This is because the number of possible polling sites, 3 , is greater than WT. Now suppose that at some more later time, say $\mathrm{t} 2$, a site failure of SN4 unfortunately occurred due to unpredictable disconnection by battery problem.

In this case, although there exist two operational sites, which are $\{\mathrm{SN} 1, \mathrm{SN} 5\}$, unfortunately any node is not allowed to formulate write quorum. This is because the number of possible polling sites, 2 , is less than WT. This dismal phenomenon continues until at least one failed node is recovered. In case the sensor node was down due to low battery, it is impossible to recover automatically without manual recovery. Consequently, every node in the sensor network is completely denied to access $x$.

In this case, all the submitted transactions to dispatch the replicated data may be blocked or aborted. Therefore, overall trans-action performance of the sensor network shall degrade substantially.

\subsection{INEFFICIENT COORDINATION IN POLLING PROCESS}

The main overhead factor occurred when the traditional QC schemes are applied to sensor network environment is that all sensor nodes are equally treated. As compared to weak nodes with almost dead battery, strong nodes with sufficient battery are far much reliable. Those strong sensor nodes are rarely dis-connected from quorum group, while those weak sensor nodes are easy to be dead or disconnected. Therefore, it is unreasonable to treat all sensor nodes equally in sensor network environment. Although base station or gateway node with reliable power source can be treated as a sort of strong nodes, this paper focused only on normal sensor nodes with limited battery source and narrow bandwidth channel.

As compared to weak sensor nodes, strong sensor nodes are even more steadily connected to quorum group, and thus they are ready to actively participate in polling process. If strong sensor nodes are allowed to possess much higher polling rights than weak sensor nodes, the strong nodes could contribute to enhance the overall polling performance substantially. 


\section{RELIABLE REPLICATION FOR IOT NETWORKS}

\subsection{SENSOR NODE MODEL}

The RC models $[8,13,14,15]$ are not sufficient to efficiently handle the excessive site failures in sensor network environment. This is because sensor network has two major differences as follows.

Frequent Sensor Node Failures: Due to the limited battery capacity, sensor nodes are easy to be disconnected intentionally (sleep/doze mode for battery energy saving) or unpredictably (exhausted battery). Due to the tiny size of the sensor node, it is vulnerable to various disasters including physical damages such as loss and robbery.

Limited Bandwidth: Since the effective transfer rate is severely limited and the data channel is shared with the other sensor nodes, the deliverable bandwidth per node would be even lower. Thus, the cost of the replica management overhead is definitely high due to the lower transfer rate.

In order to overcome these limitations and achieve high performance and high data availability, a new RC model which is far more efficient than the traditional models is strongly needed.

\subsection{PROPOSED RC SCHEME FOR IOT SENSOR NETWORK}

In order to increase transactional performance and data availability in the fault-prone sensor networks, a new RC scheme called Sensor Delegation Consensus (SDC) was proposed. The main idea of SDC is based on viable deputy polling.

4.2.1 VIABLE DEPUTY POLLING: As mentioned previously, the quorum agreement of QC scheme needs a lot of message exchanges between participating sites. Moreover, some sensor nodes are down or sleeping due to battery problem during the message transmission.

However, SDC scheme can optimize the transfer overheads by quorum-access coordination through viable deputy polling so that a small delegated group is allowed to possess much higher polling rights than normal sensor nodes. Therefore, in proportion to the weight of participating strong sensor nodes, the message overhead is gradually reduced, and overall transaction performance are gradually enhanced.

The weight related to the strong nodes could be assigned to each sensor node depending on the relative importance and reliability to the entire nodes in the replication group, and all sites are assumed to know the predefined value of weight in advance.

In SDC scheme, the sensor node which acquires more than half of the predefined delegation number has the exclusive privilege to process any transactions in the sensor network. Unlike traditional QC schemes in which all node participates in polling process, SDC exploits predefined deputy group to achieve efficient message exchanges. The deputy group consists of only strong nodes which have sufficient battery power to guarantee reliable communication for efficient polling process. For example, (Figure 3), in case deputy quorum consist of 7 strong nodes in 100 sensor network, SDC needs four message exchanges as com-pared to 51 nodes in traditional QC scheme. 


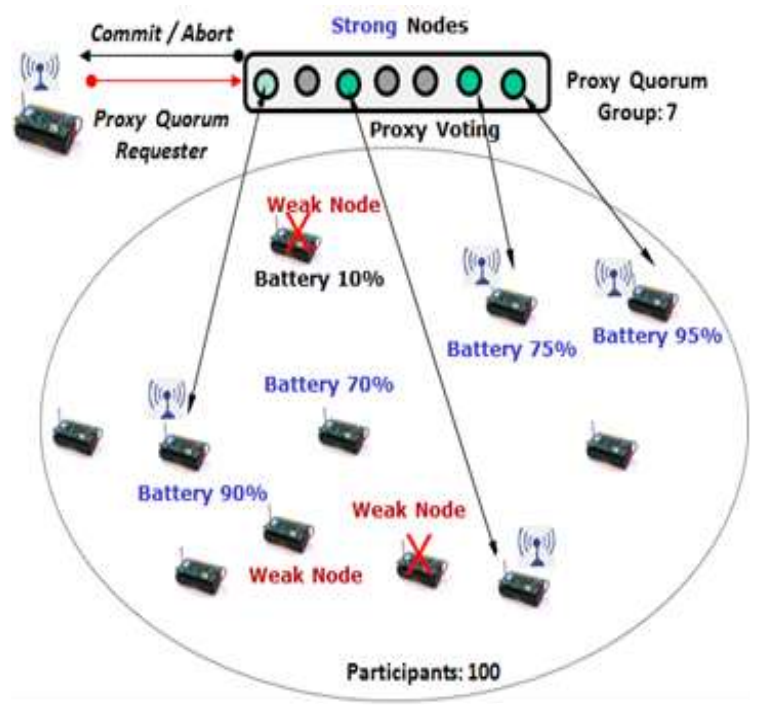

Fig. 3 Architecture of Sensor Delegation Consensus Scheme

4.2.2. MEASURING VIABILITY AND QUORUM REFORMATION: In order to maintain reliable quorum management, SDC replica-tion control scheme monitors the sensor node's viability of quorum group via the base station(or gateway) in the wireless network. The viability of each sensor node can be estimated by the level of remaining battery power. Each sensor node periodi-cally notify their energy level with strength of wireless channel. In general, the voltage of battery is detected as eight bits value in the range of 0 to 5 volts. The viability status can be sent to the gateway node when a sensor node needs to transmit sensor status to monitoring server. The viability information can be transferred rather infrequently (about twice per hour). If a cer-tain sensor node notifies low viability, quorum manager may replace the weak sensor node with the other strong sensor node to maintain reliable quorum group.

\subsection{REPLICATION CONTROL FOR SDC}

SDC includes three management modules, (1) sensor node transaction manager (SNTM), (2) sensor node synchronizing manager (SNSM) and (3) sensor node polling manager (SNPM) in each sensor node. SNTM handles read or write transactions issued by sensor node applications, i.e., from initial job to the final job. For executing the transactions in a distributed fashion, SNTM can communicate with other SNTMs. SNPM is responsi-ble for coordinating quorum-agreement process.

\subsubsection{TRANSACTION CONTROL ALGORITHM FOR SNTM}

Algorithm 1. Transaction control for sensor node

Procedure SensorNodeTransactionManager:SNTM

In: sensor transaction $\mathrm{S}$;

Out: abort or commit messages;

Begin

/*1. Do Polling Request */

PollingResult $=$ Call SensorNodePollingManager $(\mathrm{S})$;

/*2. Decide Commit or Abort */

if $($ PollingResult $=\mathrm{OK})$ then \{ 
DoCommit S;

\}

else \{

DoAbort S;

\}

end_if;

End

End of Procedure

\subsubsection{POLLING CONTROL ALGORITHM FOR SNPM}

Algorithm 2. Managing sensor node polling: SNPM

Procedure SensorNodePollingManager:

In: sensor transaction $\mathrm{S}$;

Out: latest version copy, return message;

Begin

/*1. Polling Phase */

DeputyNodes $=\mathrm{U}\{\mathrm{Di} \mid$ replica of $\mathrm{S}$ is stored at deputy node $\mathrm{Di}\}$;

DeputyQuorumThreshold $=[($ dnum +1$) / 2)\rfloor$;

AffirmativeNodes $=$ GetAffirmativeVotes $($ DeputyNodes $)$;

if ( No_ $_{-}$AffirmativeNodes)

$<$ DeputyQuorumThreshold ) then

Call_SNTM (ABORT_MESSAGE, AffirmativeNodes);

return false;

end_if ;

switch (OpType of S) \{ /*2. Operation Phase */

case DQ_READ:

Call_SNTM (COMMIT_MESSAGE, \{AffirmativeNodes\});

return true with the recent copy;

case DQ_WRITE:

for all $\mathrm{DNi} \in\{$ AffirmativeNodes $\}$ do;

Call_SNTM (WRITE_MESSAGE, DNi);

end_for;

Call_SNTM (COMMIT_MESSAGE, \{AffirmativeNodes\});

return true;

\}

End; 
Legend-

dnum: number of deputy nodes;

DN: Deputy Node;

GetAffirmativeVotes(d): get affirmative votes from set d;

Call_SNTM(M, N) call message M to Transaction Manger for Sensor Node at Node N.

\subsection{SYNCHRONIZATION MANAGEMENT FOR SENSOR NODES}

The role of SNSM is to control replica access and its locks whenever it receives a user's transaction request.

In order to control locks in SNSM, SDC make use of three lock modes: (1)Read, (2)Reserve, and (3)Write. These three lock modes will be referred hereafter as ReadLock, RsvLock, and WriteLock. The compatibility table related to the three modes is described in Table II.

Table II. Three lock modes in SNSM

\begin{tabular}{|c|c|c|c|}
\hline Requester Holder & ReadLock & RsvLock & WriteLock \\
\hline ReadLock & $\mathrm{Y}$ & $\mathrm{N}$ & $\mathrm{N}$ \\
\hline RsvLock & $\mathrm{N}$ & $\mathrm{N}$ & $\mathrm{N}$ \\
\hline WriteLock & $\mathrm{N}$ & $\mathrm{N}$ & $\mathrm{N}$ \\
\hline
\end{tabular}

In case of the write operation, the sensor node of write group should get RsvLock before they can be applied safely. The actual write operation is slow process which handles a number of sensor nodes via unstable wireless network. Thus, in proportion to the ratio of write request, transaction throughputs and responsiveness is severely degraded because of the long lock-holding time.

In order to support perfect termination of long-duration transactions, sensor nodes related to the write operation should be con-firmed to update before the physical operations. SDC achieves this by setting RsvLock for later enforcement of the physical write in the sensor nodes. After confirming RsvLock, the RsvLock is escalated to WriteLock and then the physical up-dates are enforced in the sensor node. The lock control algorithm for SNSM is described in Algorithm 3.

Algorithm 3. Handling replica access operations

Procedure Handling Sensor Node Synchronization: SNSM

\section{BEGIN}

DO \{

WAIT(msg);

CASE (msg.type) \{

IF READ:

if ( (lock mode is compatible) (no lock held) )

then 
Set_Lock(Read-Lock, msg.data);

Call_DM (DM_READ);

return READ_ACK with data sent by Data Manger;

else

insert msg into lock queue and wait;

end_if;

IF RSV:

if ( (lock mode is compatible) (no lock held) )

then

Set_Lock(RSV-Lock, msg.data);

return GET_RSV_ACK;

else

insert msg into lock queue and wait;

end_if;

IF WRITE:

if ( (lock mode is compatible) (no lock held) )

then

Set_Lock(Write-Lock, msg.data);

Call_DM(DM_WRITE);

return WRITE_ACK;

else

insert msg into lock queue and wait;

end_if;

IF COMMIT:

Release_Lock(msg.data);

return COMMIT_ACK;

IF ABORT:

Release_Lock(msg.data);

return ABORT_ACK;

;

END_CASE;

\} WHILE (true); 
END

Legend-

Set_Lock(1, d): set 1 lock on data d;

Release_Lock(d): unlock d;

Call_DM(o): Send o to DM(Data Manager);

\section{SIMULATION AND EVALUATION}

In order to verify the superior performance of SDC model, computer simulation system was developed using CSIM[16] language. The replication management systems used in this simulation experiment are proposed SDC system and GQC system.

GQC-based systems are traditional replicated data management system that can operate in the presence of site failures and net-work partition failures. In the case of a network partitioning failure, the major quorum group of large network partition with many active sites will continue to operate, while the minor group of the other small network partitions should wait until the partition failure is entirely recovered.

\subsection{SIMULATION MODEL}

The main simulation model and the transaction processing architectures are based on the queuing system as shown in Figure 4.

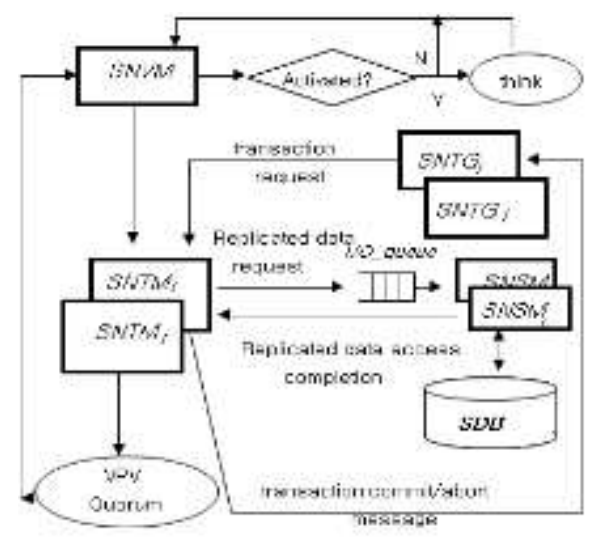

Legend-

SNTG: Sensor Node Transaction Generator

SNSM: Sensor Node Synchronization Manager

SNTM: Sensor Node Transaction Manager

SNPM: Sensor Node Polling Manager

SDB: Sensor Database

Fig. 4 Architecture of CSIM simulation model

The default queuing model used was the closed queuing model provided by the CSIM, which included transaction generation queues, various transaction processing units, data I/O queues, and network transmission queues for each sensor node.

The simulation system consists of a transaction generator of sensor nodes for accessing replicated data (SNTG), a data synchronization manager for accessing replicated data (SNSM), a sensor node polling manager(SNPM), and a sensor node trans-action manager (SNTM).

The sensor node transaction generator generates sensor transactions at a certain interval with exponential distribution. Transaction managers manage the performance of 
transactions from start to finish, and call SNPM for requested access to replicated data. When a SNPM is called, it performs an access authorization procedure for the relevant replica, SNSM performs synchronization, and returns the results to the transaction manager.

Major performance evaluation indicators for the CSIM simulations are sensor transaction throughput, abort ratio and average response time of sensor transactions. The sensor transaction throughput means how many sensor transactions were processed per second.

The sensor transaction abort ratio means the percentage of the sensor transactions that were cancelled in the course of the sensor transactions processing.

Each transactions processing scheme has two variant polices, called abort and retry, depending on what was done if a certain transaction failed to access replicated copy. If SDC uses the policy of abort-oriented action, it aborts the given transaction after waiting until the predefined time limit is over.

The major simulation parameters are the number of sensor nodes, the renewal rate, site failure rate, and the replication ratio. The number of sensor nodes devices has changed from 200 to 800 units.

The renewal rate, which is the ratio of write transactions to the number of read or write transactions, is set to a typical criterion of $25 \%$. The replication ratio, which represents the number of replication data objects for the total number of objects, was specified in terms of $5 \%$ to $20 \%$. The above set figures are fixed values but can be changed as needed in the course of the experiment.

\subsection{RESULTS AND ANALYSIS}

The simulations were conducted with the two replication management schemes and focused on major parameters that had performance-sensitive effects. The revision ratio for individual data objects was set to $25 \%$ by default.

From the preliminary tests, it is observed that the response time of the SDC was significantly different from that of others. This means that the SDC have a large transient portion. Therefore, for simplicity, the transient phase of read and write transactions below 100 was removed. The moving average response time is presented in Figure 5.

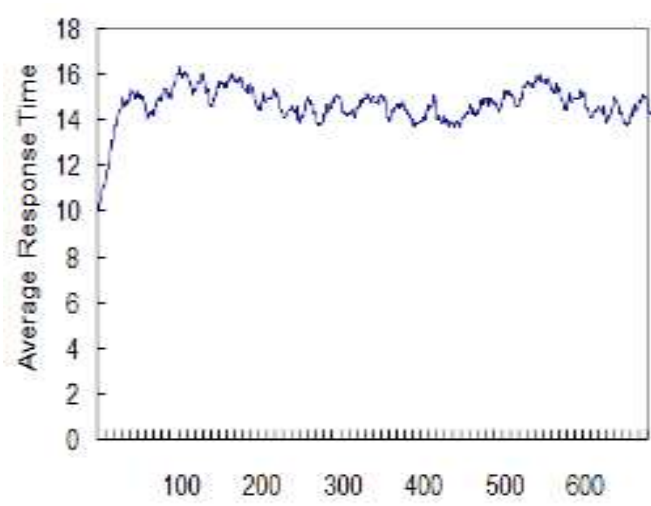

Fig. 5 Moving average response time in CSIM Simulation

This experiment is designed to analyze how the number of node failures affects the performance of replication management systems. Figure 6 presents the transaction throughput, and Figure 7 presents the average response time of the transaction.

As the frequency of site failures increases, the rate of transaction withdrawal gradually goes up because of the quorum over-heads of the weak sensor nodes. In addition, overall transaction processing performance was measured and SDC was superior to GQC. 
In Figure 6, the transaction performance of each replication management system has been reduced as the number of site failures exceeds approximately 5 . This means that site failure is a major factor that significantly affects performance, and enabling transactions further leads to performance degradation.

However, even under the same conditions, the proposed SDC technique was higher than the GQC technique. This is because the deputy polling method reduces excessive message overhead for replicated data, thereby reducing the transaction withdrawal rate associated with the replicated data. Consequently, the response time of SDC is relatively low as compared to GQC. In Figure 7, when the number of site failures reaches 5, the GQC technique was heavily influenced by the quorum formation overhead caused by message transfer delay.

Note that there is a significant performance difference between the retry-oriented and abort-oriented policy for GQC. The former represents about 1.5 times slower execution than the latter. This is why the retry-oriented policy makes GQC retry the failed transaction uselessly, while abort-oriented policy makes GQC cancel the failed transaction and handle the following transactions.

However, the two transaction handling policies cannot be com-pared in a sense that they are quite different approaches to handle the failed transaction caused by the site failures in sensor networks. That is, the amount of lost and recovery work de-pends substantially on the detailed policy actually taken.

\begin{tabular}{|c|c|c|c|c|}
\hline \multirow[t]{2}{*}{ T_SF } & \multicolumn{4}{|c|}{ Throughput } \\
\hline & GaC-Retry & GQC:Abort & SDC-Retry & SDC-Abort \\
\hline 3 & 12.11 & 12.74 & 14.08 & 15.08 \\
\hline 4 & 9.22 & 10.34 & 13.55 & 13. \\
\hline 5 & .99 & 9.76 & 13.62 & 12.8 \\
\hline 6 & 5.89 & 9.45 & 12.67 & 13. \\
\hline 7 & 4.87 & 9.60 & 12.72 & 13.4 \\
\hline 8 & 4.32 & 9.33 & 13.62 & 12. \\
\hline 9 & 3.81 & 9.27 & 13.37 & 12.7 \\
\hline 10 & 3.46 & 9.48 & 12.63 & 13.3 \\
\hline 11 & 3.07 & 9.45 & 13.64 & 13. \\
\hline 12 & 2.81 & 9.35 & 13.19 & 13. \\
\hline
\end{tabular}

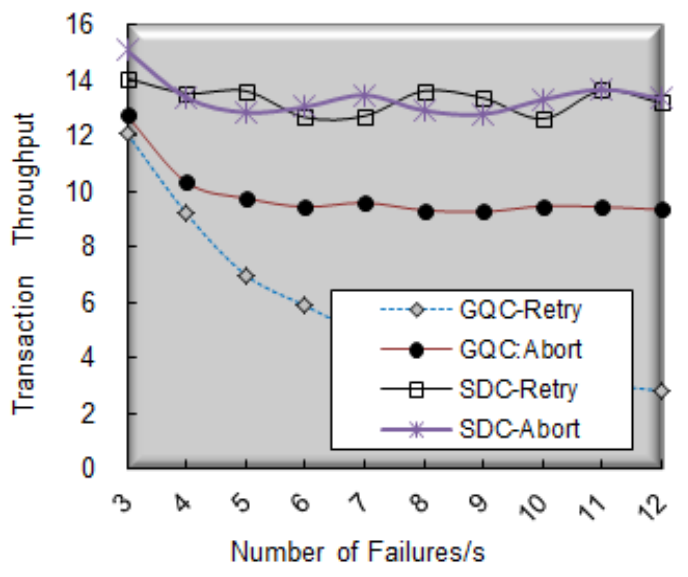

Fig. 6 Transaction throughput in CSIM simulation

\begin{tabular}{|c|r|r|r|r|}
\hline \multirow{2}{*}{ T_SF } & \multicolumn{4}{|c|}{ Avg_Response_Time } \\
\cline { 2 - 5 } & GQC-Retry & GRC:Abort & SDC-Retry & SDC-Abort \\
\cline { 2 - 5 } 3 & 8.26 & 7.85 & 7.1 & 6.63 \\
\hline 4 & 10.85 & 9.67 & 7.38 & 8.07 \\
\hline 5 & 14.3 & 10.25 & 7.34 & 7.78 \\
\hline 6 & 16.99 & 10.58 & 7.89 & 7.66 \\
\hline 7 & 20.54 & 10.42 & 7.86 & 7.42 \\
\hline 8 & 23.15 & 10.72 & 7.34 & 7.74 \\
\hline 9 & 26.28 & 10.79 & 7.48 & 7.82 \\
\hline 10 & 28.9 & 10.55 & 7.92 & 7.51 \\
\hline 11 & 32.59 & 10.58 & 7.33 & 7.31 \\
\hline 12 & 35.56 & 10.7 & 7.58 & 7.49 \\
\hline
\end{tabular}

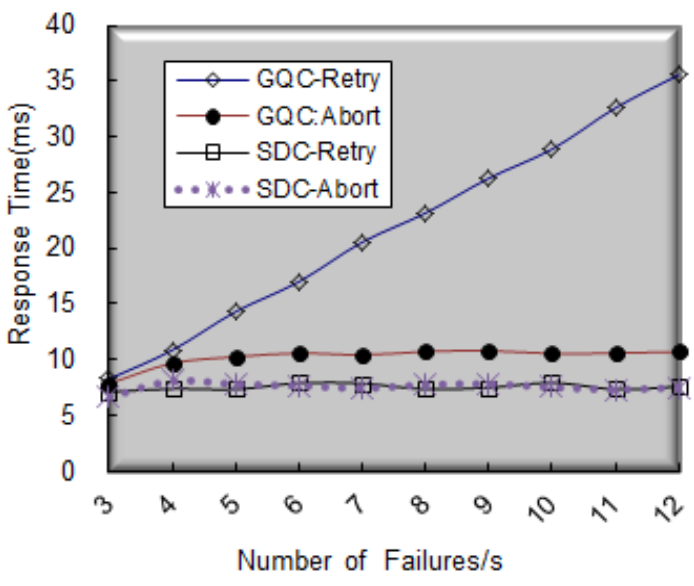

Fig. 7 Average response time in CSIM Simulation 
On the contrary, the performance difference between the two handling policies in SDC is considered to be insignificant. As mentioned above, this is why SDC activated most of the blocked transactions by using deputy quorum before abort time limit. In the respect of the sensor transaction throughput and response time, it is concluded that SDC scheme outperforms traditional QC-based replication control schemes.

\section{CONCLUSION}

In order to reliably control multi-copy consistency in the sensor network systems, a new replica management model named sensor delegation consensus was proposed on the basis of the general quorum consensus algorithm.

In order to lessen the performance degradation caused by excessive node failures in fault-prone IoT sensor database environment, sensor delegation consensus scheme exploits the notion of viable deputy polling which makes the best use of reliable sensor nodes in polling process.

To show the efficiency of the proposed model, CSIM simulation system was also developed. Since sensor delegation consensus scheme has a generic functionality of faulttolerant and efficient replication management, the proposed model can be used in the various IoT sensor network environments.

\section{ACKNOWLEDGEMENT}

This research was supported by Basic Science Research Pro-gram through the National Research Foundation of Korea (NRF) funded by the Ministry of Education (2018R1D1A1B07044418).

\section{REFERENCES}

[1] Yong Yao, Johannes Gehrke, "Query Processing for Sensor Net-works," IEEE Pervasive Computing, Vol.3, No.1, (2004), pp. 46-55. (March 2004)

[2] Plale. B., "Using Global Snapshots to Access Data Streams on the Grid", Lecture Notes in Computer Science, Vol.3165, pp.191-201, 2004

[3] Javier J. M., Colonna J. G., Soares R. B., Figueiredo C. M. S., and Nakamura E. F., "Compressive Sensing for Efficiently Collecting Wildlife Sounds with Wireless Sensor Networks", International Conference on Computer Communications and Networks (ICCCN), (2012), pp.1-7.

[4] Bernstein P., Hadzilacos V., and Goodman N., Concurrency con-trol and recovery in database systems, Addison-Wesley Press, (1987).

[5] Kumar, "A. Hierarchical quorum consensus: a new algorithm for managing replicated data," IEEE Trans. Comput., Vol.40, No.9, (1991), pp.996-1004.

[6] Spasojevic, M. and Berman, P., "Voting as the optimal static pes-simistic scheme for managing replicated data, IEEE Trans. Parallel Distrib. Syst.,Vo. 5, No.1, (1994), pp. 64-73.

[7] Herlihy, M., "A quorum-consensus replication method for abstract data types," ACM Trans. Comput. Syst., Vol.4, No.1, (1986), pp.32-53.

[8] Lin, X., "A fully distributed quorum consensus method with high fault-tolerance and low communication overhead," Theor. Comput. Sci., Vol. 185, No. 2, (1997), pp.259-275.

[9] Malkhi, D. and Reiter, M., "Byzantine quorum systems," Distrib. Comput., Vol.11, No.4, (1998), pp.203-213.

[10] M. Vukolic., "Quorum Systems: With Applications to Storage and Consensus," Synthesis Lectures on Distributed Computing Theory. Morgan \& Claypool (2012).

[11] Edson Tavares de Camargo, Elias P. Duarte Jr., Fernando Pedonez: "A Consensus-based Fault-Tolerant Event Logger for High Perfor-mance Applications," Euro-Par 2017: Parallel Processing, (2017), pp.415-427.

[12] Cachin C. and Vukolic M., "Blockchain consensus protocols in the wild," Technical Report arXiv:1707.01873, IBM Research - Zurich (July 2017).

[13] Bernstein, P. and Goodman, N.: "An algorithm for concurrency control and recovery in replicated distributed databases," ACM Trans. Database Syst., Vol.9, No. 4, (1984), pp.596-615.

[14] Ittai Abraham, Dahlia Malkhi, Kartik Nayak, Ling Ren, and Alex-ander Spiegelman.: "Solidus: An incentive-compatible cryptocur-rency based on permissionless byzantine consensus," arXiv preprint (2016). 
International Journal of Advanced Science and Technology

Vol.124 (2019)

[15] Pass R. and Shi E., "Hybrid consensus: Efficient consensus in the permissionless model," Cryptology ePrint Archive, Report 2016/917, (2016).

[16] CSIM, "Introduction to CSim Modeling Environment", (2018), available online: http://www.csim.com/overview.html. 